\title{
EFEITOS POSITIVOS DE UMA INTERVENÇÃO POR INTEGRAÇÃO CURRICULAR NA PROMOÇÃO DA AUTORREGULAÇÃO DA APRENDIZAGEM
}

\author{
MARIA ANTÔNIA ROMÃO DA SILVA ${ }^{1}$ \\ ORCID: https://orcid.org/0000-0003-3557-8702 \\ PAULA MARIZA ZEDU ALLIPRANDINI ${ }^{2}$ \\ ORCID: https://orcid.org/0000-0003-4677-4258
}

\begin{abstract}
RESUMO: Este artigo apresenta como temática a promoção da autorregulação da aprendizagem. Teve como objetivo verificar os efeitos de uma intervenção por integração curricular na autorregulação da aprendizagem junto a estudantes do curso de Design de Moda de uma Instituição Pública do Paraná. Caracterizada como uma pesquisa quase-experimental, foi dividida em três fases: pré-teste; intervenção; e pós-teste. No pré-teste, participaram 84 alunos do $1^{\circ}$ ao $4^{\circ}$ ano. 22 participaram do programa de intervenção e 60 alunos do pós-teste. Para a coleta de dados, foi aplicada a Escala de Avaliação de Estratégias de Aprendizagem. Os resultados apontaram para um aumento na frequência no uso das estratégias pelos participantes da intervenção em relação aos 3 fatores da escala: Autorregulação Cognitiva e Metacognitiva, Autorregulação dos Recursos Internos e Contextuais e Autorregulação Social, o que indicou a possibilidade de promover um estudante mais autorregulado em situação de aprendizagem por meio do ensino do uso de estratégias de aprendizagem.
\end{abstract}

Palavras-chave: Autorregulação da aprendizagem, Ensino Superior, estratégias de aprendizagem.

\section{POSITIVE EFFECTS OF AN INTERVENTION BY INTEGRATED CURRICULUM IN THE PROMOTION OF SELF-REGULATION OF LEARNING}

\begin{abstract}
This paper has as theme the promotion of self-regulation in learning. It had as objective verifying the effects of an intervention by integrated curriculum in self-regulation of learning envolving students of the course of Fashion Design of a Public Institution in Paraná, Brazil. Characterized as a quasi-experimental research, it was divided into three stages: pre-testing; intervention; and post-testing. In the pre-test 84 students from 1st to 4 th years took part. 22 took part of the intervention program and 60 students participated in post-testing. For data collection it was used the Scale of Learning Strategies. The results point to an increase of using the strategies by students that participated in the intervention regarding 3 factors of the scale: Cognitive and Meta-cognitive Self-regulation, Self-regulation of Internal and Contextual Resources and Social Self-regulation, indicating the possibility of promoting more selfregulated students in learning situation by teaching the use of learning strategies.
\end{abstract}

\footnotetext{
${ }^{1}$ Univerisade Estadual de Londrina (UEL). Londrina, PR, Brasil. <maria.antonia.romao@gmail.com>

${ }^{2}$ Univerisade Estadual de Londrina (UEL). Londrina, PR, Brasil. < paulaalliprandini@uel.br> Educação em Revista|Belo Horizonte|v.36|e227164|2020
} 
Keywords: Self-regulation of learning, Higher Education, learning strategies.

\section{EFECTOS POSITIVOS DE UNA INTERVENCIÓN DE PROGRAMAS INTEGRADOS PARA PROMOVER LA AUTORREGULACIÓN DEL APRENDIZAJE}

RESÚMEN: Este artículo trata de la promoción de la autorregulación del aprendizaje. Su objetivo ha sido verificar los efectos de una intervención de programas integrados en la autorregulación del aprendizaje con estudiantes del curso de Diseño de Moda de una Institución Pública en el estado de Paraná, Brasil. La investigación es de carácter cuasiexperimenta y se ha dividido en tres fases: pre test, intervención y post test. En el pre test, participaron 84 estudiantes del $1^{\circ}$ al $4^{\circ}$ curso, 22 participaron en el programa de intervención y 60 estudiantes en el post test. Para la recolección de datos se utilizó la Escala de Evaluación de Estrategias de Aprendizaje. Los resultados apuntan a un aumento en el uso de las estrategias por parte de los estudiantes que participaron en la intervención, con respecto a los 3 factores de la escala: autorregulación cognitiva y metacognitiva, autorregulación de recursos internos y contextuales y autorregulación social. Todo lo anterior demuestra la posibilidad de promover un estudiante más autorregulado en situación de aprendizaje, enseñando el uso de estrategias de aprendizaje.

Palabras clave: Autorregulación del aprendizaje, enseñanza superior, estrategias de aprendizaje. 


\section{INTRODUÇÃO}

Com base nas reflexões acerca do projeto do curso de Design na área de Moda, somado às reflexões e aos estudos sobre aprendizagem no contexto educativo, tendo como referencial teórico a Psicologia Cognitiva, baseada na Teoria do Processamento da Informação, este artigo apresenta uma pesquisa de Mestrado do tipo quase experimental realizada com o objetivo de verificar os efeitos de uma intervenção por integração curricular nos processos de autorregulação da aprendizagem em estudantes do curso de Design de Moda de uma Instituição Pública do Paraná.

Estudos voltados para o processo de aprendizagem dos métodos de projeto de Design são necessários, com destaque para questões gerais como as especificidades do ensino de Design - que exige o desenvolvimento da capacidade de autogestão pelo estudante e um ensino interdisciplinar -, a não adequação dos métodos lineares e exatos às atuais necessidades do ato projetivo (CELASCHI; MORAES, 2013; LAWSON, 2011), bem como questões específicas do curso estudado, como a taxa de evasão e reprovação nos primeiros anos (RISSI; MARCONDES, 2013) e dificuldades dos discentes observadas no cotidiano educacional, como problemas para: gerenciar informações; definir objetivos; estabelecer ligação entre os conteúdos (MIOTTO, 2014; SANCHES, 2016).

Profundas transformações nas relações estabelecidas entre o indivíduo e o cenário dinâmico contemporâneo influenciam diretamente o contexto educativo, imponto novos desafios aos envolvidos no processo de ensino e aprendizagem, exigindo do indivíduo uma postura autônoma, reflexiva e ativa de agente do próprio saber, o que reverbera na prática entre educador e educando. A promoção dos processos de autorregulação no período de escolarização se apresenta como meio favorável para o desenvolvimento do pensamento crítico-reflexivo (BORUCHOVITCH, 2014; KARABENICK; DEMBO, 2011; SAMPAIO; POLYDORO; ROSÁRIO, 2012; VEIGA SIMÃO; FRISON, 2013; ZIMMERMAN; SCHUNK, 2011).

O estudo buscou contribuir diretamente com o ambiente acadêmico do Curso de Design de Moda pesquisado ao lançar olhar sobre a promoção da autorregulação da aprendizagem no contexto educativo por meio da prática pedagógica realizada na disciplina de ensino de projeto.

A autorregulação é um processo autodirecionado inerente ao ser humano, que pode ser compreendido como meio pelo qual o indivíduo coloca em prática suas habilidades para autogerir ações e exercer controle sobre recursos internos e ambientais, bem como sobre níveis cognitivos e emocionais em prol de metas prefixadas. O papel emancipador exercido pela autorregulação no contexto educacional vem sendo sugerido por estudiosos de diferentes linhas teóricas com a elaboração de diversas abordagens da Psicologia e modelos de autorregulação da aprendizagem desde meados da década de 1980, com o objetivo de investigar meios de tornar o educando mais autônomo e autoconsciente ao longo do processo de ensino e aprendizagem para melhor controlar os recursos internos e externos em função das suas necessidades e tarefa de aprendizagem (ZIMMERMAN, 2013; ZIMMERMAN; KITSANTAS, 2014).

Para a Teoria do Processamento de Informação, a autorregulação da aprendizagem e os processos autorregulatórios se dão por meio da prática constante de planejamento e uso de estratégias em benefício do fluxo de informação, para facilitar a organização dos conhecimentos em redes significativas, contribuindo com o processo de aquisição, retenção e resgate do conhecimento. Nessa perspectiva, a aprendizagem se apoia em três aspectos: os dados produzidos por diversos canais presentes no ambiente; o papel ativo do estudante; e a exposição do conhecimento processado (BZUNECK; 2010), com destaque aos processos cognitivos empregados pelo indivíduo ao receber e reelaborar a informação.

Oportunizar a aprendizagem autorregulada exige uma postura pedagógica que considere as particularidades de cada aprendizagem ao longo do processo, favorecendo o aprimoramento da autorregulação por meio de ações que permitam ao educando desenvolver a capacidade de autogestão, planejamento e controle consciente das próprias ações, de modo que se possa elaborar e empregar estratégias de aprendizagem em favor das metas acadêmicas individuais.

Desse modo, a promoção da autorregulação da aprendizagem, em nível universitário no ensino de Design, apresenta subsídios frutíferos para aprimorar a capacidade de aprender dos estudantes do Ensino Superior de Design de Moda, e encontra na Psicologia Cognitiva, sob a lente do Processamento da Informação, aporte teórico sólido. 


\section{APRENDIZAGEM AUTORREGULADA: O USO DE ESTRATÉGIAS E PROGRAMAS DE INTERVENÇÃO}

O constructo da autorregulação da aprendizagem é um processo autodirecionado, que envolve procedimentos internos de nível cognitivo, metacognitivo, motivacional e externos que devem ser adaptados pelo estudante de modo consciente a cada tarefa de aprendizagem. O uso deliberado das estratégias de aprendizagem é considerado característica principal da aprendizagem autorregulada e exerce papel fundamental no processo (BORUCHOVITCH; SANTOS, 2015; DEMBO; SELI, 2004; KARABENICK; DEMBO 2011; POZO, 2002; ZIMMERMAN; SCHUNK, 2011), garantindo uma aprendizagem eficaz e o sucesso acadêmico, pois desafia o educando a assumir um papel ativo e autônomo, tornando-o autor e gestor da sua aprendizagem ao criar condições favoráveis para que ela ocorra de forma efetiva.

As estratégias de aprendizagem podem ser compreendidas como técnicas integradas que o estudante utiliza para facilitar a aquisição, retenção e recuperação das informações (DEMBO, 1994) que envolvem estratégias cognitivas e metacognitivas, variáveis motivacionais, sentimentos e comportamentos compatíveis com a aprendizagem (WEINSTEIN; ACEE; JUNG, 2011). Seu uso requer postura ativa do discente para mobilizar recursos e conhecimentos que favoreçam o aprendizado de modo estratégico, além de um vasto repertório de estratégias de aprendizagem, visto que estas possuem particularidades e devem ser aplicadas em função das metas e caráter das atividades acadêmicas.

Em diferentes níveis educacionais, os programas de intervenção se apresentam como opções eficientes na promoção de práticas pedagógicas que favorecem a aprendizagem autorregulada por meio da ampliação do repertório de estratégias em benefício da formação de estudantes mais estratégicos e autoconscientes (BORUCHOVITCH, 2010; GANDA, 2016; TEIXEIRA; ALLIPRANDINI, 2013; SANTOS, 2016; STEINER, 2016).

Para atingir o objetivo de promover a autorregulação da aprendizagem, o programa deve contemplar uma estrutura multidimensional com componentes cognitivos, metacognitivos e motivacionais (BORUCHOVITCH; COSTA; NEVES, 2005). Os programas de intervenção promotores da autorregulação da aprendizagem podem apresentar dois formatos distintos: 1) integração curricular (integrated programs) (HOFER; YU; PINTRICH, 1998; GANDA, 2016) ou infusão curricular (ROSÁRIO; POLYDORO, 2012), o qual incorpora ao contexto da disciplina instruções referente ao uso das estratégias de aprendizagem; 2) sobreposição curricular (courses adjunct) (HOFER; YU; PINTRICH, 1998; GANDA, 2016), designada por Rosário e Polydoro (2012) como justaposição curricular em que as ações promotoras do ensino e o uso de estratégias e aprendizagem autorreguladas não estão inseridos no currículo, mas em horário extraclasse. O presente estudo destaca o formato de integração curricular (integrated programs) que, segundo os autores citados anteriormente, apresenta maior probabilidade de promover processos autorregulatórios.

\section{PERCURSO METODOLÓGICO}

Inicialmente, o projeto tramitou e foi aprovado pelo Comitê de Ética em pesquisa Envolvendo Seres Humanos, em 10 de agosto de 2016, conforme parecer 1.670.907.

O presente estudo trata de uma pesquisa do tipo quase-experimental ${ }^{3}$, desenvolvida junto aos estudantes do curso de Design de Moda da Instituição pesquisada e foi estruturado em três etapas, conforme segue: 1 ) pré-teste realizado com discentes da $1^{\mathrm{a}}$. a $4^{\mathrm{a}}$. séries do curso no segundo semestre do ano letivo de 2016; 2) intervenção no formato de integração curricular vinculada ao conteúdo da disciplina anual de Metodologia do Projeto, realizada com estudantes da $2^{\text {a }}$ série do curso ao longo do primeiro semestre letivo de 2017 ; 3 ) pós-teste junto aos alunos da $2^{a}$. a $4^{a}$. séries ao término do primeiro semestre letivo de 2017.

\footnotetext{
${ }^{3}$ Neste método, não é possível controlar todas as fontes de distorção, nem realizar uma seleção aleatória dos participantes, mas permite analisar causa e efeito de um fenômeno e o delineamento de grupos não equivalentes (BOAVENTURA; FARIA, 2006; GIL, 2008).
} 
Realizada on-line, a etapa de pré-teste ocorreu entre 20 e 29 de setembro de 2016, com a aplicação coletiva do instrumento Escala de Estratégias de Aprendizagem para Estudantes Universitários (EEA-U) (BORUCHOVITCH; SANTOS, 2015), pela plataforma Google Forms, durante o período de aula de cada série.

Participaram do pré-teste 84 estudantes da $1^{\mathrm{a}}$ a $4^{\mathrm{a}}$ séries do curso de Design de Moda que aceitaram colaborar com a pesquisa, distribuídos por série da seguinte forma: 21 estudantes da $1^{a}$ série (25\%), 21 matriculados na $2^{\mathrm{a}}$ série (21\%), 23 da $3^{\mathrm{a}}$ série $(27,4 \%)$, e 19 da $4^{\mathrm{a}}$ série do curso $(22,6 \%)$. Os participantes da $1^{\text {a }}$. série foram considerados como grupo experimental - sendo que, no momento da intervenção, encontravam-se matriculados na $2^{\text {a }}$. série -, os das demais séries foram considerados como do grupo controle (63 estudantes).

Para a realização do pós-teste no ano letivo de 2017, participaram 60 discentes distribuídos entre a $2^{a}$ série com 22 participantes (36,66\%), $3^{a}$ série com $16(26,68 \%)$ e $4^{a}$ série com 22 (36,66\%). Não participaram da fase de pós-teste os estudantes da $3^{\text {a }}$ série que trancaram a matrícula no ano letivo de 2017 e os estudantes que estavam matriculados na $4^{a}$ série no ano letivo de 2016, uma vez que, no ano de realização do pós-teste, já eram egressos do curso.

$\mathrm{Na}$ Figura 1, apresentada a seguir, é possível observar as características de cada etapa da pesquisa.
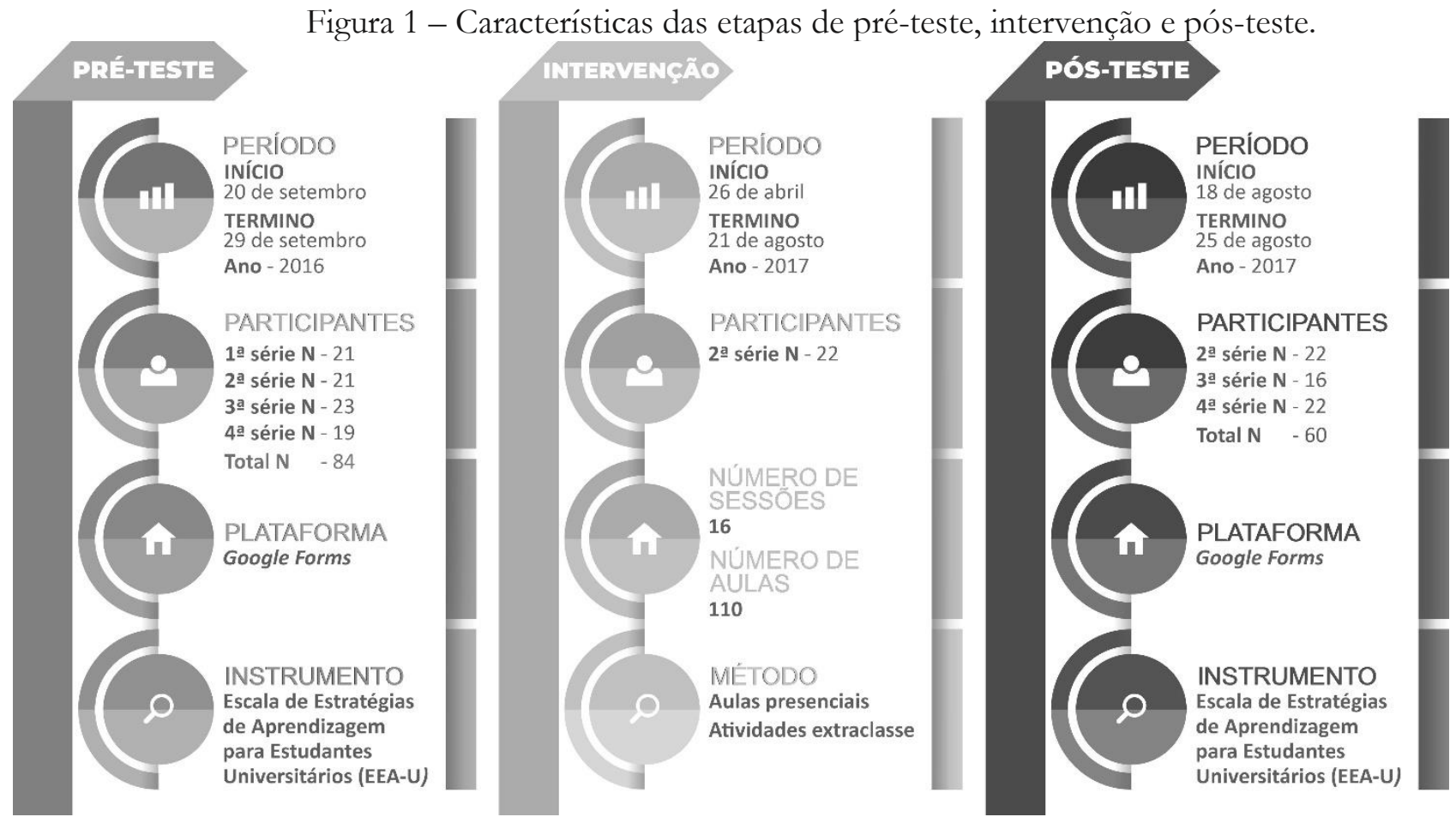

Fonte: Elaborado pelas autoras (2019).

O programa de intervenção pedagógica foi realizado entre 26 de abril e 21 de agosto de 2017, segundo o formato de Integração Curricular em que o professora/pesquisadora incorporou instruções do uso de estratégias de aprendizagem intencionalmente no contexto da disciplina anual de Metodologia do Projeto, contemplando as estratégias de aprendizagem apresentadas como menos frequentes, tendo como base os resultados obtidos em situação de pré-teste pelos estudantes participantes matriculados na $1^{\text {a }}$ série no ano letivo de 2016 que se encontravam matriculados na $2^{a}$ série do curso em 2017, no momento da intervenção.

Como apontado na Figura 1, o programa de intervenção foi realizado ao longo de 16 sessões, divididas em 9 sessões no primeiro bimestre e 7 no segundo, totalizando 110 aulas presenciais. Em alguns casos, as sessões apresentaram mais de um dia de aula por semana, com número de duas ou mais aulas de 50 minutos, inteirando 91 horas e 40 minutos de trabalho em sala, mais atividades extraclasse. Durante 
o processo de intervenção, foi incentivado o uso das estratégias autorregulatórias de gerenciamento de tempo, planejamento, sociais, contextuais, emocionais e motivacionais.

As sessões contemplaram atividades práticas, orientações e tarefas autorreflexivas. Os estudantes foram orientados a montar individualmente cronogramas semanais planejando futuras ações, levados a desenvolver trabalhos, estudar em grupo e discutir os conteúdos com os colegas. Para facilitar o uso de algumas estratégias - como pedir auxílio em caso de dúvidas -, foi criado um canal de comunicação via Facebook para estudantes e docente.

$\mathrm{Na}$ terceira etapa, após a intervenção, foi realizado o pós-teste, no qual foi reaplicada a Escala de Estratégias de Aprendizagem para Estudantes Universitários (EEA-U) no formato on-line pela plataforma Google Forms. A aplicação do instrumento foi realizada coletivamente, ao término do $1^{\circ}$ semestre letivo de 2017, junto aos estudantes da $2^{a}$ série $(n=22)$, na penúltima sessão da intervenção. Com os discentes das $3^{a}$ e $4^{a}$ séries $(\mathrm{n}=38$ ), a aplicação do pós-teste foi realizada no período de 20 a 25 de agosto, de modo não presencial.

Para verificar do nível de autorregulação de aprendizagem por meio da frequência do uso de estratégias de aprendizagem pelos estudantes nas etapas de pré-teste e pós-teste, foi utilizada a Escala de Estratégias de Aprendizagem para Estudantes Universitários (EEA-U) (BORUCHOVITCH; SANTOS, 2015). Trata-se de uma escala do tipo Likert de quatro pontos (1 a 4), composta por 35 itens de múltipla escolha - com quatro alternativas de resposta (sempre, às vezes, raramente e nunca) e 1 questão opcional.

A escala é estruturada em três fatores: Fator 1 - Autorregulação Cognitiva e Metacognitiva com 23 itens no total, dentre os quais pode-se citar como exemplo: escrever com suas palavras o que entendeu do texto; 2 - Autorregulação dos Recursos Internos e Contextuais com 8 itens, como: manter a calma diante de tarefas difíceis; Fator 3 - Autorregulação Social com 4 itens, tendo como exemplo: pedir ajuda em caso de dúvidas.

\section{PROCEDIMENTO DE ANÁLISE DOS DADOS}

Foi atribuída pontuação às respostas dos participantes, segundo a alternativa selecionada, seguindo as orientações da escala EEA-U: sempre $=4$; às vezes $=3$; raramente $=2$; nunca $=1$. No item 26, a pontuação é invertida, ou seja, sempre $=1$, às vezes $=2$, raramente $=3$ e nunca $=4$ pontos. Para análise dos dados da escala obtidos no pré-teste e pós-teste, empregou-se procedimentos de estatística descritiva ao calcular a frequência (\%), média, o desvio padrão e a análise inferencial com a análise da consistência interna por meio do Alpha de Cronbach, análise de variância e teste de Tukey, quando os dados apresentaram diferenças estatisticamente significativas $(\mathrm{p}<0,05)$ entre as séries e fases de pré e pós-teste. Para tanto, foram utilizados o software Excel e Statistic.

\section{RESULTADOS E DISCUSSÃO}

Com o propósito de verificar os efeitos da intervenção pedagógica realizada junto aos alunos da $2^{\text {a }}$. série do Curso de Design de Moda quanto à promoção da autorregulação, por meio do ensino de estratégias de aprendizagem, foram comparados os resultados obtidos nas etapas de pré-teste e pós-teste.

Inicialmente, verificou-se a consistência do instrumento sobre os itens comtemplados nos fatores, extraindo coeficiente aceitável do alpha de Cronbach, indicando que os dados coletados permitem interpretações seguras. Fase de pré-teste: Fator 1 - Autorregulação Cognitiva e Metacognitiva $(\alpha=0,80)$; Fator 2 - Autorregulação dos Recursos Internos e Contextuais $(\alpha=0,80)$ e Fator 3 - Autorregulação Social $(\alpha=0,60)$. Situação de pós-teste: Fator 1 - Autorregulação Cognitiva e Metacognitiva $(\alpha=0,90)$; Fator 2 - Autorregulação dos Recursos Internos e Contextuais $(\alpha=0,70)$ e Fator 3 - Autorregulação Social $(\alpha=0,70)$.

$\mathrm{Na}$ sequência, foram analisados os dados obtidos em situação de pré-teste e pós-teste da amostra total para verificar o nível de autorregulação da aprendizagem dos universitários do Curso de Design de Moda quanto ao uso das estratégias de aprendizagem, no que tange a autorregulação cognitiva e metacognitiva, autorregulação dos recursos internos e contextuais e autorregulação social.

É possível observar, na Figura 2, a Média, o Desvio Padrão (Dp), valores mínimos e máximos da amostra total em situação de pré-teste e pós-teste, a partir dos autorrelatos dos estudantes 
do Curso de Design de Moda, mediante a aplicação da Escala de Estratégias de Aprendizagem para Estudantes Universitários (EEA-U).

Figura 2 - Média, Valor mínimo e máximo e Desvio padrão (Dp) da Amostra Total em situação de pré-teste $(\mathrm{n}=84)$ e pós-teste $(\mathrm{n}=60)$

\begin{tabular}{|c|c|c|c|c|c|c|c|c|}
\hline & \\
\hline & & PRÉ- & TESTE & & & Pós- & TESTE & \\
\hline FATORES & MÉDIA & $\begin{array}{l}\text { VALOR } \\
\text { MÍNIMO }\end{array}$ & $\begin{array}{l}\text { VALOR } \\
\text { MÁXIMO }\end{array}$ & DP & MÉDIA & $\begin{array}{l}\text { VALOR } \\
\text { MÍNIMO }\end{array}$ & $\begin{array}{l}\text { VALOR } \\
\text { MÁXIMO }\end{array}$ & DP \\
\hline $\begin{array}{l}\text { AUTORREGULAÇÃO } \\
\text { COGNITIVAE } \\
\text { METACOGNITIVA }\end{array}$ & 3,01 & 2,09 & 3,83 & 0,31 & 3,08 & 1,71 & 3,88 & 0,43 \\
\hline $\begin{array}{l}\text { AUTORREGULAÇÃO DOS } \\
\text { RECURSOS INTERNOS E } \\
\text { CONTEXTUAIS }\end{array}$ & 2,80 & 1,38 & 4,00 & 0,53 & 2,86 & 1,25 & 4,00 & 0,55 \\
\hline $\begin{array}{l}\text { AUTORREGULAÇÃO } \\
\text { SOCIAL }\end{array}$ & 2,73 & 1,75 & 4,00 & 0,50 & 2,91 & 1,50 & 4,00 & 0,64 \\
\hline
\end{tabular}

Fonte: Elaborado pelas autoras (2019).

Tendo como base os dados apresentados pela amostra total em situação de pré-teste, é possível observar que as estratégias do Fator 3 - Autorregulação Social (2,73) - apresentaram a menor média de utilização, seguido pelo Fator 2 - Autorregulação dos Recursos Internos e Contextuais, que apresentou uma média intermediária $(2,80)$. O Fator 1 - Autorregulação Cognitiva e Metacognitiva apresentou maior média $(3,01)$. Os dados do pós-teste demonstram aumento na média de todos os fatores, principalmente no Fator $3(2,91)$.

O resultado apontado pela amostra total em situação de pré-teste corrobora com estudos anteriores (ALCARÁ; SANTOS, 2013; KARABENICK, 2004; KARABENICK; DEMBO, 2011; MOHALLEM, 2016; NEWMAN, 2000; STEFANOU et al., 2013) ao apresentar menor média de utilização para as estratégias de Autorregulação Social. Os resultados obtidos em análises realizadas nos estudos de Alcará e Santos (2013) e Mohallem (2016), em relação ao uso das estratégias de aprendizagem por universitários, evidenciam maior média para Autorregulação Cognitiva e Metacognitiva e menor para as estratégias de Autorregulação Social.

Os dados relativos ao Fator 2 (Autorregulação dos Recursos Internos e Contextuais) apontam que as estratégias voltadas para planejamento apresentaram menor frequência de uso, dados que se aproximam dos apresentados por Stefanou et al. (2013), que indicaram o baixo uso de estratégias relacionadas ao gerenciamento de tempo e do ambiente de estudo por discentes de cursos baseados em projetos. A análise também indica que os estudantes apresentam alto nível de distração durante as atividades acadêmicas, com destaque para o item distrair-se ou pensar em outra coisa quando está lendo, estudando ou fazendo os trabalhos $(1,51)$.

Dentre as estratégias de autorregulação social apresentadas no Fator 3, as de estudar em grupo $(1,90)$ e discutir a matéria com os colegas para ver se entendeu $(2,00)$ foram as que apresentaram a menor frequência de uso. Entre as estratégias do Fator 2 (Autorregulação dos Recursos Internos e Contextuais), foram: administrar seu tempo de estudo (2,65); manter a calma diante de tarefas dificeis $(2,76)$ e planejar suas atividades de estudo (2,80). As estratégias relacionadas ao Fator 1 (Autorregulação Cognitiva e Metacognitiva) apresentaram a maior média. Porém, as seguintes estratégias: criar perguntas sobre o assunto que está estudando e tentar respondê-las $(1,65)$; recorrer a outros textos e livros sobre o assunto $(2,26)$; ler textos complementares, além dos indicados pelo professor $(2,38)$ e pedir auxílio ao professor sobre as dúvidas na matéria $(3,00)$ apresentaram, segundo relato dos participantes, baixa frequência. 
A Figura 3, apresentada a seguir, evidencia as médias, os respectivos desvios padrão e a variância relativa ao uso das estratégias de aprendizagem por série, de acordo com cada fator da escala em situação de pré-teste e pós-teste.

Figura 3 - Comparação das Médias, Desvio padrão (dp) e variância obtidos por fator da escala e série dos estudantes em fase de pré-teste e pós-teste.



Fonte: Elaborado pelas autoras (2019).

Por meio da análise de variância aplicada em função das séries às quais os participantes estavam matriculados, os dados indicam que não houve diferença significativa no uso das estratégias entre as séries em situação de pré-teste, o que sugere, apesar de não ter sido um estudo longitudinal, que os estudantes não se tornaram mais estratégicos e autorregulados ao longo da formação acadêmica.

Enquanto que, na análise dos dados, considerando os dados da fase de pós-teste, as diferenças foram estatisticamente significativas $(p<0,05)$ entre as séries em relação aos fatores contemplados pela escala.

O teste Tukey indicou que, em relação ao Fator 1 (Autorregulação Cognitiva e Metacognitiva), houve diferenças estatisticamente significativas entre a $2^{\mathrm{a}}, 3^{\mathrm{a}}$ e $4^{\mathrm{a}}$ séries. Já para o Fator 2 (Autorregulação dos Recursos Internos e Contextuais) e Fator 3 (Autorregulação Social), os dados dos participantes da $2^{a}$ série, que vivenciaram a intervenção pedagógica, indicaram diferenças estatisticamente 
significativas em relação aos dados dos estudantes das $3^{\text {a }}$. e $4^{\text {a }}$. séries, não havendo diferenças significativas entre os estudantes destas duas últimas.

Os dados apontaram um aumento na frequência em relação ao uso das estratégias da aprendizagem pelos estudantes da $2^{a}$ série no pós-teste, sendo que os estudantes das demais séries apresentaram uma diminuição na frequência, o que indicou um aumento no nível de autorregulação da aprendizagem pelos estudantes da $2^{a}$. série, considerando os fatores propostos pela Escala. Ao comparar os resultados obtidos pelos participantes da intervenção, os estudantes apresentaram as seguintes médias, respectivamente, na situação de pré e pós-teste: Fator 1 - Autorregulação Cognitiva e Metacognitiva (3,03 e 3,46), Fator 2 - Autorregulação dos Recursos Internos e Contextuais $(2,95$ e 3,27) e Fator 3 Autorregulação Social $(2,79$ e 3,52).

Os resultados do grupo de Controle em situação de pré-teste e pós-teste apontaram para um baixo nível do uso de estratégias importantes para o processo de aprendizagem autorregulada que precisam ser ensinadas aos estudantes, uma vez que há dificuldade em resistir a distrações, bem como a dificuldade em gerenciar o tempo, fatores estes que podem interferir no processo de ensino aprendizagem, principalmente ao considerar que o curso de Design de Moda preconiza uma formação acadêmica baseada no desenvolvimento de projetos.

Além disso, de modo geral, observou-se a preferência por estratégias superficiais que consomem menos esforço e tempo e o baixo uso de estratégias de aprendizagem profunda, como, por exemplo, elaborar perguntas sobre o assunto que está estudando e tentar respondê-las. $\mathrm{O}$ uso de estratégias superficiais resulta na retenção da informação para reprodução fatual posterior, não destinada a estabelecer conexões profundas do conteúdo a ser processado, sendo o processo de aprendizagem conduzido por uma motivação extrínseca balizada no resultado final da tarefa, por vezes distante do verdadeiro ato de aprender (DUARTE, 2004; ROSÁRIO et al., 2010), resultando em experiências acadêmicas negativas que conduzem os estudantes à desistência e reprovação (DUARTE, 2004; ROSÁRIO et al., 2010).

A diminuição na média do uso das estratégias de aprendizagem, apresentada no pós-teste pelos estudantes que não participaram do programa de intervenção sugere a necessidade de verificar as práticas pedagógicas desenvolvidas no curso. Segundo Galvão, Cãmara e Jordão (2012), os estudantes apresentam experiências educacionais prévias, hábitos de estudo já estabelecidos e carência no repertório de estratégias, o que influencia diretamente o processo de aprendizagem. Contudo, os discentes não são os únicos personagens responsáveis por sanar as lacunas, o docente e o discente compartilham da responsabilidade do resultado do processo de aprendizagem (BIGGS; KEMBER; LEUNG, 2001).

Diversos fatores interferem no uso efetivo das estratégias, como: aspectos motivacionais; atribuição de significado à tarefa; conhecimentos prévios; repertório; natureza do conteúdo ensinado; métodos de ensino e avaliação; e contexto institucional. Desse modo, os resultados indicam que o contexto educativo no qual os estudantes estão inseridos favorece as concepções de aprendizagem que, não apenas estimula o uso de estratégias de abordagem superficiais, como os impele a empregarem cada vez menos as estratégias autorregulatórias.

A partir dos resultados obtidos, ao comparar as situações de pré-teste e pós-teste, é possível inferir que os estudantes participantes do programa de intervenção apresentaram alta no nível de autorregulação em relação às outras séries, por apresentarem aumento na média no que tange os três fatores presentes na Escala, enquanto os estudantes das séries que não vivenciaram a intervenção apresentaram uma diminuição na frequência em relação ao uso das estratégias.

Para melhor verificar os efeitos do programa de intervenção na promoção da autorregulação, foram calculadas as médias obtidas pelos participantes quanto ao uso das estratégias de aprendizagem trabalhadas ao longo da intervenção pedagógica, em situação de pré-teste e pós-teste. Por meio da análise de variância, foi verificada diferença estatisticamente significativa entre as médias obtidas no pré e pós teste $(p<0,05)$ para os três fatores da escala. Mediante a aplicação do Teste Tukey, foi possível verificar em quais itens da escala se encontram as diferenças significativas para cada fator. A Figura 4 apresenta as médias obtidas nas situações de pré e pós-teste para cada item da escala relativos aos Fatores 1, 2 e 3. 
Figura 4 - Médias e variância das respostas na fase de Pré-teste e Pós-teste relativas aos Fatores 1,2 e 3.

\begin{tabular}{|c|c|c|}
\hline $\begin{array}{l}\text { FATOR } 1 \\
\text { ITENS }\end{array}$ & $\begin{array}{c}\text { MÉDIA } \\
\text { PRÉ-TESTE }\end{array}$ & $\begin{array}{c}\text { MÉDIA } \\
\text { PÓS-TESTE }\end{array}$ \\
\hline 4. Resumir os textos indicados para estudo. & 2,81 & 2,82 \\
\hline 5. Ler os textos indicados pelo professor. & 3,24 & 3,27 \\
\hline 7. Escrever com suas palavras o que entendeu do texto. & 3,00 & 3,00 \\
\hline $\begin{array}{l}\text { 8. Ler textos complementares, além dos indicados pelo } \\
\text { professor. }\end{array}$ & 2,24 & $3,64^{*}$ \\
\hline $\begin{array}{l}\text { 9. Elaborar perguntas e respostas sobre o assunto } \\
\text { estudado. }\end{array}$ & 1,43 & $3,14^{*}$ \\
\hline 13-Pedir auxílio ao professor sobre as dúvidas na matéria. & 3,10 & $3,59 *$ \\
\hline 20-Recorrer a outros textos e livros sobre o assunto. & 2,57 & $3,55^{*}$ \\
\hline $\begin{array}{l}\text { 30-Fazer algum esquema no papel (esboço, gráfico ou } \\
\text { desenho) para melhor entender as relações entre eles. }\end{array}$ & 2,95 & $3,73^{*}$ \\
\hline 34-Reler a matéria para entendê-la melhor. & 3,48 & $3,77^{*}$ \\
\hline $\begin{array}{l}\text { 35-Criar perguntas sobre o assunto que está estudando } \\
\text { e tentar respondê-las. }\end{array}$ & 1,62 & $3,00 *$ \\
\hline $\begin{array}{l}\text { FATOR } 2 \\
\text { ITENS }\end{array}$ & $\begin{array}{c}\text { MÉDIA } \\
\text { PRÉ-TESTE }\end{array}$ & $\begin{array}{c}\text { MÉDIA } \\
\text { PÓS-TESTE }\end{array}$ \\
\hline 17-Administrar seu tempo de estudo. & 2,86 & $3,55^{*}$ \\
\hline 18-Organizar seu ambiente de estudo. & 3,05 & 3,27 \\
\hline 19-Manter a calma diante de tarefas difíceis. & 2,86 & 3,00 \\
\hline 21-Planejar suas atividades de estudo. & 2,95 & $3,59 *$ \\
\hline $\begin{array}{l}\text { 26-Distrair-se ou pensar em outra coisa quando está } \\
\text { lendo, estudando ou fazendo os trabalhos. }\end{array}$ & 1,52 & $2,68^{*}$ \\
\hline $\begin{array}{l}\text { FATOR } 3 \\
\text { ITENS }\end{array}$ & $\begin{array}{c}\text { MÉDIA } \\
\text { PRÉ-TESTE }\end{array}$ & $\begin{array}{c}\text { MÉDIA } \\
\text { PÓS-TESTE }\end{array}$ \\
\hline 16-Pedir ajuda aos colegas em caso de dúvidas. & 2,52 & $3,91^{*}$ \\
\hline 28-Estudar em grupo. & 1,90 & $3,77^{*}$ \\
\hline $\begin{array}{l}\text { 32-Discutir a matéria com os colegas para ver } \\
\text { se entendeu. }\end{array}$ & 3,29 & $3,91^{*}$ \\
\hline
\end{tabular}

Fonte: Elaborado pelas autoras (2019).

Educação em Revista|Belo Horizonte|v.36|e227164|2020 
Ao comparar as médias obtidas nas fases de pré e pós-testes dos itens do Fato 1 que foram trabalhados na intervenção, conforme pode ser observado, o teste Tukey indicou diferenças estatisticamente significativas para os itens $8,9,13,20,30,34$ e 35, não havendo diferença estatisticamente significativa nos itens 4,5 e 7 .

Quanto às médias das respostas para os itens do Fator 2, elas evidenciam um aumento em relação ao uso de todas as estratégias, com destaque ao item 26. O Teste Tukey indicou diferenças estatisticamente significativas para os itens 17, 21 e 26, enquanto nos itens 18 e 19 não houve diferenças significativas entre as médias. Quanto ao Fator 3, é possível observar diferença estatisticamente significativa para todos os itens trabalhados ao longo da intervenção.

Portanto, essa análise em relação às médias obtidas corrobora com os resultados apresentados anteriormente, ao comparar os dados do pré e pós-teste por meio da frequência a partir das opções de respostas e análise de variância relativa ao uso das estratégias de aprendizagem por série.

Quanto aos resultados obtidos neste estudo, relativos à análise estatística entre as médias do uso da estratégia pedir ajuda ao professor e da Autorregulação Social na situação de pós-teste, é possível conferir um efeito positivo quanto ao aumento na frequência do uso das estratégias em decorrência da intervenção considerado favorável para o processo de aprendizagem autorregulada. Segundo Newman (2000), o educando autorregulado apresenta repertório específico como: habilidades cognitivas e compreensão, o que permite, ao mesmo tempo, perceber quando precisa de auxílio, como solicitar a ajuda e pontuar a informação necessária; habilidades sociais e compreensão, sabendo reconhecer quem pode ajudar e saber como realizar o pedido apropriado; recursos afetivos e motivacionais que contemplam objetivos, crença de autoeficácia, atitudes e sentimentos que envolvem tolerância com o nível de dificuldade da tarefa, desejo de interação social e determinação. Desse modo, o estudante apresenta consciência das próprias limitações que o impedem de atingir seus objetivos de forma independente.

Em relação às estratégias autorregulatórias de gerenciamento de tempo e planejamento, os resultados obtidos no pós-teste indicam alta na frequência do uso dessas estratégias pelos estudantes que participaram do programa de intervenção. O planejamento para realização das atividades acadêmicas, automonitoramento e autoavaliação são compreendidas como estratégias essenciais para um processo de aprendizagem bem-sucedido, estreitamente guiado por metas e objetivos que determinam o conjunto de estratégias a serem empregadas para atingir o fim proposto (KARABENICK; DEMBO, 2011). Da Silva Gusmão (2011) salienta que a estratégia de organizar e planejar de forma eficiente o uso do espaço e o tempo disponível contribui para uma aprendizagem significativa.

Foi expressiva a mudança na frequência referente às estratégias autorregulatórias motivacionais e emocionais, pois os itens manter a calma diante de tarefas dificeis e distrair-se ou pensar em outra coisa quando está lendo, estudando ou fazendo os trabalhos apresentaram dados quantitativos positivos. Contudo, a análise de variância indicou diferença estatisticamente significativa apenas no item referente ao controle de distratores, mesmo havendo aumento na média do uso da estratégia voltada para o domínio das emoções, o que indica a necessidade de continuar a trabalhar essa estratégia. Possíveis fatores que contribuíram para alteração foram abordados por Karabenick e Dembo (2011) acerca de aspectos relacionados ao uso efetivo das estratégias de aprendizagem, como o desenvolvimento de crenças de autoeficácia e autopercepções positivas, o uso de estratégias autorregulatórias, como o planejar e estudar em grupo e estabelecer como meta o aprender.

Isto posto, os resultados obtidos no presente estudo corroboram com outras pesquisas que indicam resultados positivos na promoção do uso de estratégias autorregulatórias por meio de programas de intervenção pedagógica em diferentes níveis de ensino (BORUCHOVITCH, 2010; FERNANDES; FRISON, 2015; GANDA, 2016; SANTOS, 2016; TEIXEIRA; ALLIPRANDINI, 2013; STEINER; 2016). Segundo Boruchovitch e Santos (2015), o uso das estratégias auxilia no controle de aspectos pessoais e ambientais. Portanto, cabe ao docente favorecer o aprimoramento do repertório de estratégias de aprendizagem ao incorporar instruções acerca das mesmas para que os estudantes consigam transferir o uso dos procedimentos para diferentes situações acadêmicas. Promover aprendizagem autorregulada 
apresenta-se como um objetivo educacional necessário a fim de facilitar o processamento da informação pelo aprendiz.

\section{CONSIDERAÇÕES}

Com o objetivo de verificar os efeitos de uma intervenção por integração curricular na autorregulação da aprendizagem junto a estudantes de um curso de Design de Moda de uma Instituição Pública do Paraná, o presente estudo apresentou resultados que evidenciaram um aumento no nível de autorregulação da aprendizagem dos estudantes participantes da intervenção por integração curricular, com aumento significativo da frequência do uso de estratégias que compõe os fatores de Autorregulação Cognitiva e Metacognitiva, Autorregulação dos Recursos Internos e Contextuais e Autorregulação Social, em especial, em relação às seguintes estratégias contempladas no programa de intervenção: ler textos complementares, além dos indicados pelo professor; elaborar perguntas e respostas sobre o assunto estudado; pedir auxílio ao professor sobre as dúvidas na matéria; recorrer a outros textos e livros sobre o assunto; fazer algum esquema no papel (esboço, gráfico ou desenho) para melhor entender as relaçöes entre eles; reler a matéria para entendê-la melhor; criar perguntas sobre o assunto que está estudando e tentar respondê-las; administrar seu tempo de estudo; planejar suas atividades de estudo; distrair-se ou pensar em outra coisa quando está lendo, estudando ou fazendo os trabalhos; pedir ajuda aos colegas em caso de dúvidas; estudar em grupo; discutir a matéria com os colegas para ver se entendeu.

Isto posto, entende-se, por meio dos resultados, que a intervenção apresentou efeitos positivos ao instruir e possibilitar o uso de estratégias de aprendizagem pouco empregadas pelos estudantes participantes.

No geral, a intervenção foi voltada para o desenvolvimento do pensamento estratégico do estudante e enriquecimento do repertório de estratégias dos mesmos. Contudo, as seguintes estratégias trabalhadas ao longo do programa de intervenção não apresentaram diferença estatisticamente significativa ao comparar o pré-teste e pós-teste, mesmo com aumento na média: resumir os textos indicados para estudo; ler os textos indicados pelo professor; escrever com suas palavras o que entendeu do texto; organizar seu ambiente de estudo; manter a calma diante de tarefas difíceis. Esses resultados indicam a necessidade de rever algumas práticas adotadas durante a intervenção, de forma a possibilitar mudanças significativas quanto ao uso dessas estratégias.

Há que se considerar que a presente pesquisa apresenta limitações, uma vez que o instrumento, por basear-se em autorrelatos, obtém dados balizados no juízo dos participantes, de modo que os estudantes podem recorrer a respostas que considerem mais adequadas socialmente.

Novas investigações relacionadas às intervenções quanto ao uso de estratégias de aprendizagem ao longo do curso são necessárias, assim como de futuros estudos voltados para o favorecimento do automonitoramento e controle dos aspectos motivacionais e emocionais dos estudantes no contexto escolar.

\section{REFERÊNCIAS}

ALCARÁ, Adriana Rosecler; SANTOS, Acácia Aparecida Angeli. dos. Compreensão de leitura, estratégias de aprendizagem e motivação em universitários. Psico, v. 44, n. 3, p. 411-420, 2013. Disponível em: revistaseletronicas.pucrs.br/ojs/index.php/revistapsico/article/view/12258/10416. Acesso em: 11 set. 2018.

BIGGS, John; KEMBER, David; LEUNG, Doris Yin Ping. The revised two - factor study process questionnaire: R-SPQ-2F. British journal of educational psychology, v. 71, n. 1, p. 133-149, 2001. Disponível em: onlinelibrary.wiley.com/doi/abs/10.1348/000709901158433. Acesso em: 11 set. 2018.

BORUCHOVITCH, Evely. Autorregulação da aprendizagem: contribuições da psicologia educacional para a formação de professores. Psicologia Escolar e Educacional, v. 18, n. 3, p. 401-409, 2014. Disponível em: www.scielo.br/pdf/pee/v18n3/1413-8557-pee-18-03-0401.pdf. Acesso em: 10 set. 2018. 
BORUCHOVITCH, Evely. A autorregulação da aprendizagem e a escolarização inicial. In: BORUCHOVITCH, Evely; BZUNECK, José Aloysio. (Org.). Aprendizagem: processos psicológicos e o contexto social na escola. Petrópolis, RJ: Vozes, 2010. p. 55-88.

BORUCHOVITCH, Evely; COSTA, Elis Regina; NEVES, Edna Rosa Correia. Estratégias de aprendizagem: contribuições para a formação de professores nos cursos superiores. In: JOLY, Maria Cristina Rodrigues Azevedo; SISTO, Fermino Fernandes; SANTOS, Acácia Aparecida Angeli dos. (Org.). Questões do cotidiano universitário. São Paulo, SP: Casa do Psicólogo, 2005. p. 239-260.

BORUCHOVITCH, Evely; SANTOS, Acácia Aparecida Angeli dos. Psychometric Studies of the Learning Strategies Scale for University Students. Paidéia, Ribeirão Preto, v. 25, n. 60, p. 19-27, 2015. Disponível em: http://www.scielo.br/scielo.php?pid=S0103863X2015000100019\&script=sci_arttext\&tlng=es. Acesso em: 10 set. 2018.

BZUNECK, José Aloysio. Aprendizagem por processamento da informação: uma visão construtivista. In: BORUCHOVITCH, Evely; BZUNECK, José Aloysio. (Org.). Aprendizagem: processos psicológicos e o contexto social na escola. 2 ed. Petrópolis: Vozes, 2010. p. 17-54.

CELASCHI, Flaviano; MORAES, Dijon de. Futuro, Bem-Estar, Interdependência: Palavras-Chave para o Design Contemporâneo. In: MORAES, Dijon de; CELASCHI, Flaviano. (Org.). Caderno de Estudos Avançados em Design: Design e Humanismo. v. 7. Barbacena: EdUEMG, 2013. p. 35-60.

DA SILVA GUSMÃO, Pablo. A aprendizagem autorregulada da percepção musical no ensino superior: uma pesquisa exploratória. Opus, v. 17, n. 2, p. 121-140, 2011.

DEMBO, Myron H. Applying educational psychology. 5th ed. White Plains, NY: Longman Publishing Group. 1994.

DEMBO, Myron H.; SELI, Helena Praks. Students' resistance to change in learning strategies courses. Journal of developmental education, v. 27, n. 3, p. 2, 2004. Disponível em: eric.ed.gov/?id=EJ718559. Acesso em: 29 jul. 2018.

DUARTE, António Manuel. Auto-regulação e abordagens à aprendizagem. In: DA SILVA, Adelina Lopes; DUARTE, António Manuel; SÂ, Isabel; VEIGA-SIMÃO, Ana Margarida. (Ed.) Aprendizagem auto-regulada: Perspectivas psicológicas e educacionais. Porto: Porto Editora, 2004. p. 39-54.

FERNANDES, Verônica Rodriguez; FRISON, Lourdes Maria Bragagnolo. Estratégias de aprendizagem autorregulatória no ensino superior: escrita de um artigo científico. Revista Psicopedagogia da Educação, São Paulo, v. 41, $2^{\circ}$ sem, p. 37-49, 2015. Disponível em: pepsic.bvsalud.org/scielo.php?script=sci_arttext\&pid=S1414-69752015000200003. Acesso em: 27 jul. 2017.

GALVÃO, Afonso; CÂMARA, Jacira; JORDÃO, Michelle. Estratégias de aprendizagem: reflexões sobre universitários. Revista Brasileira de Estudos Pedagógicos, Brasilia, v. 93, n. 235, p. 627-644, 2012. Disponível em: www.scielo.br/scielo.php?pid=S2176 66812012000400006\&script=sci_abstract\&tlng=pt. Acesso em: 27 jul. 2017.

GANDA; Danielle Ribeiro. A autorregulação da aprendizagem de estudantes em curso de formação de professores: Um programa de intervenção. Campinas. 2016. 143f. Tese (Doutorado em Educação), Faculdade de Educação da Universidade Estadual de Campinas, Campinas. 2016. Disponível em: repositorio.unicamp.br/handle/REPOSIP/325460. Acesso em: 13 ago. 2017.

HOFER, Barbara; YU, Shirley; PINTRICH, Paul. Teaching college students to be self-regulated learners. In: SCHUNK, Dale; ZIMMERMAN, Barry. (Eds.). Self-regulated learning: From teaching to selfreflective practice. New York: Guilford Press. 1998. p. 57-85. 
KARABENICK, Stuart A. Perceived achievement goal structure and college student help seeking. Journal of Educational Psychology, v. 96, p. 569-581, 2004. Disponível em: psycnet.apa.org/fulltext/2004-18154-015.html. Acesso em: 15 ago. 2017.

KARABENICK, Stuart. A.; DEMBO, Myron H. Understanding and facilitating self - regulated help seeking. New Directions for Teaching and Learning, v. 2011, n. 126, p. 33-43, 2011. Disponível em: onlinelibrary.wiley.com/doi/abs/10.1002/tl.442. Acesso em: 13 ago. 2017.

LAWSON, Bryan. Como arquitetos e designers pensam. São Paulo: Oficina de Textos, 2011.

MIOTTO, Thassiana de Almeida. O ensino da metodologia projetual no desenvolvimento de produtos de moda: um estudo de caso na Universidade Estadual de Londrina. 2014. 110f. Dissertação (Mestrado) - Curso de Ppg em Design, Faculdade de Arquitetura Artes e Comunicação, Universidade Estadual Paulista Julio de Mesquisa Filho, Bauru. 2014. Disponível em: https://repositorio.unesp.br/bitstream/handle/11449/126289/000838482.pdf?sequence=1\&isAllowe d=y. Acesso em: 20 jul. 2017

MOHALLEM, Rosana Maria Martins. Estratégias de aprendizagem em universitários: avaliação e intervenção. 2016. 130f. Tese (Doutorado em Psicologia) - Universidade São Francisco, Itatiba, 2016. Disponível em: https:/ /www.usf.edu.br/galeria/getImage/427/17279032934113009.pdf. Acesso em: 22 set. 2017.

NEWMAN, Richard S. Social influences on the development of children's adaptive help seeking: the role of parents, teachers, and peers. Developmental review, v. 20, n. 3, p. 350-404, 2000. Disponível em: https://www.sciencedirect.com/science/article/pii/S0273229799905026. Acesso em: 27 jul. 2017.

POZO, Juan Ignacio. Teorias cognitivas da aprendizagem. 3. ed. Porto Alegre: Artes Médicas, 2002.

RISSI, Marinalva Calabrez; MARCONDES, Marta Ap. Santana. Reflexão sobre a reprovação, retenção e evasão na UEL: retenção em 2010, reprovação 2010 a 2012 e evasão 2003 a 2012 nos cursos de graduação. Londrina: Universidade Estadual de Londrina, 2013. Disponivel em: http://www.uel.br/proplan/aval-institucional/Reflexao-Reprovacao-Evasao-na-UEL-Livro-05-122013.pdf. Acesso em: 20 jul. 2017.

ROSÁRIO, Pedro; POLYDORO, Soely Aparecida Jorge. Capitanear o aprender: promoção da autorregulação da aprendizagem no contexto escolar. São Paulo: Casa do Psicólogo. 2012.

ROSÁRIO, Pedro et. al. Enhancing self-regulation and approaches to learning in first-year college students: a narrative-based programme assessed in the Iberian Peninsula. European Journal Psychology Education, n. 25, p. 411-428, fev. 2010. Disponível em: https://link.springer.com/article/10.1007/s10212-010-0020-y. Acesso em: 28 jul. 2017.

SAMPAIO, Rita Karina Nobre; POLYDORO, Soely Aparecida Jorge; ROSÁRIO, Pedro. Autorregulação da aprendizagem e a procrastinação acadêmica em estudantes universitários. Cadernos de Educação, Pelotas, n. 42, p. 119-142, mai. 2012. Disponível em: https://repositorium.sdum.uminho.pt/handle/1822/28087. Acesso em: 19 ago. 2017.

SANCHES, Maria Celeste de Fatima. O projeto do intangível na formação de designers de moda: repensando as estratégias metodológicas para a sintaxe da forma na prática projetual. 2016. 252f. Tese apresentada à Faculdade de Arquitetura e Urbanismo da Universidade de São Paulo e à Universitat Politècnica de València para obtenção do título de Doutor por ambas as instituições, conforme Convenio Acadêmico Internacional para Dupla Titulação de Doutorado. Universidade de São Paulo, São Paulo, 2016. Disponível em: http://www.teses.usp.br/teses/disponiveis/16/16134/tde-16022017-094603/ptbr.php. Acesso em: 15 ago. 2017. 
SANTOS, Deivid Alex dos. Efeito de uma intervenção em estratégias de aprendizagem em estudantes de biologia do ensino médio. 2016. 146f. Dissertação (Mestrado em Educação) Universidade Estadual de Londrina, Londrina, 2016. Disponível em: http://www.uel.br/pos/mestredu/images/stories/downloads/dissertacoes/2016/2016__SANTOS_Deivid_Alex.pdf. Acesso em: 20 ago. 2017.

STEFANOU, Candice; et al. Self-regulation and autonomy in problem-and project-based learning environments. Active Learning in Higher Education, v. 14, n. 2, p. 109-122, 2013. Disponível em: ttps://journals.sagepub.com/doi/abs/10.1177/1469787413481132?journalCode=alha. Acesso em: 10 jul. 2017.

STEINER, Hillary H. The Strategy Project: Promoting Self-Regulated Learning through an Authentic Assignment. International Journal of Teaching and Learning in Higher Education, v. 28, n. 2, p. 271-282, 2016. Disponível em: http://www.isetl.org/ijtlhe/pdf/IJTLHE2211.pdf. Acesso em: 15 ago. 2017.

TEIXEIRA, Andrea; ALLIPRANDINI, Paula Maria Zedu. Intervenção no uso de estratégias de aprendizagem diante de dificuldades de aprendizagem. Psicologia Escolar e Educacional, v. 17, n. 2, 2013. Disponível em: http://www.scielo.br/scielo.php?script=sci_arttext\&pid=S141385572013000200010. Acesso em: 21 jul. 2017.

VEIGA SIMÃO, Ana Margarida; FRISON, Lourdes Maria. Autorregulação da aprendizagem: abordagens teóricas e desafios para as práticas em contextos educativos. Cadernos de Educação, n. 45, p. 2013. 20, Disponivel em: periodicos.puccampinas.edu.br/seer/index.php/reveducacao/article/view/299. Acesso em: 19 ago. 2017.

WEINSTEIN, Claire Ellen; ACEE, Taylor W.; JUNG, Jaehak. Self-regulation and learning strategies. New directions for teaching and learning, v. 2011, n. 126, p. 45-53, 2011. Disponível em: https://onlinelibrary.wiley.com/doi/abs/10.1002/tl.443. Acesso em: 19 ago. 2017.

ZIMMERMAN, Barry. J. From cognitive modeling to self-regulation: a social cognitive career path. Educational psychologist, v. 48, n. 3, p.135-147, 2013. Disponível em: www.tandfonline.com/doi/abs/10.1080/00461520.2013.794676. Acesso em: 20 ago. 2017.

ZIMMERMAN, Barry J.; SCHUNK, Dale H. Handbook of self-regulation of learning and performance. New York, NY: Taylor \& Francis, 2011.

ZIMMERMAN, Barry J.; KITSANTAS, Anastasia. Comparing students' self-discipline and selfregulation measures and their prediction of academic achievement. Contemporary Educational Psychology, v. 39, n. 2, p. 145-155, 2014. Disponível em: www.sciencedirect.com/science/article/pii/S0361476X14000162. Acesso em: 20 ago. 2017.

Submetido: 09/09/2019

Aprovado: $22 / 02 / 2020$ 